\title{
Hormonal activity in clinically silent adrenal incidentalomas
}

Anna Babińska, Małgorzata Siekierska-Hellmann, Krzysztof Błaut, Anna Lewczuk, Piotr Wiśniewski, Maria Gnacińska, Łukasz Obołończyk, Renata Świątkowska-Stodulska, Krzysztof Sworczak

Department of Endocrinology and Internal Diseases, Medical University of Gdansk, Poland

Submitted: 31 August 2010

Accepted: 7 November 2010

Arch Med Sci 2012; 8, 1: 97-103

DOI: 10.5114 /aoms.2012.27288

Copyright @ 2012 Termedia \& Banach

\section{Abstract}

Introduction: The rapid development of modern imaging techniques, has led to an increase in accidentally discovered adrenal masses without clinically apparent hormonal abnormalities. Such tumours have been termed "incidentalomas". The diagnostic work-up in patients with adrenal incidentalomas is aimed at the determination of hormonal activity of the tumour and identification of patients with potentially malignant tumours. The aim of our study was a retrospective analysis of selected clinical characteristics and hormonal studies in accidentally discovered adrenal tumours.

Material and methods: Fourty hundred sixty-three patients with serendipitously discovered adrenal masses, diagnosed and treated in the Department of Endocrinology and Internal Diseases, Medical University of Gdansk as well as in the affiliated Endocrinology Clinic between 1993 and October of 2009 were included in the analysis. Out of all patients, 245 were referred for adrenalectomy. Results: We found that clinically "silent" tumours often demonstrate subclinical hormonal activity. In our report, increased 24-h urinary excretion of cortisol correlated positively with tumour size $(p<0.001)$. Moreover, a statistical relationship was demonstrated between tumour size and serum cortisol concentration assessed in the $1 \mathrm{mg}$ dexamethasone suppression test $(p<0.001)$. Increased values of dehydroepiandrosterone/dehydroepiandrosterone sulphate were more often found in malignant than in benign tumours $(p<0.01)$. Urinary concentrations of 17-ketosteroids correlate positively with diagnosis of adrenocortical cancer $(p=0.02)$.

Conclusions: We found that clinically "silent" tumours often demonstrate subclinical hormonal activity (subclinical Cushing syndrome, subclinical pheochromocytoma, low-symptomatic adrenocortical cancer).

Key words: adrenal incidentaloma, subclinical Cushing syndrome, subclinical Conn syndrome, subclinical pheochromocytoma, low-symptomatic adrenocortical cancer.

\section{Introduction}

An incidentally discovered adrenal tumour (incidentaloma) is a mass in one or both adrenal glands detected accidentally during an imaging study performed for other reasons [1-3].

By definition, incidentalomas do not have prominent clinical symptoms but can demonstrate low-level activity of the respective hormones, termed sub- or preclinical syndromes. The first step in the diagnostic work-up of adrenal incidentalomas is the assessment of their hormonal activity [4-7].

\author{
Corresponding author: \\ Anna Babińska MD \\ Department of Endocrinology \\ and Internal Medicine \\ Medical University of Gdansk \\ Debinki 7, 80-952 Gdansk, \\ Poland \\ Phone: + 48583492840 \\ Fax: + 48583492841 \\ E-mail: a.mail@wp.pl
}


There have been several attempts at determining the usefulness of individual laboratory assays in the work-up of adrenal incidentalomas [4, 6, 8].

The aim of our study was to perform a retrospective analysis of selected clinical characteristics and hormonal studies in accidentally discovered adrenal tumours. The study intended to establish parameters that could be used to determine the need for surgery or clinical follow-up only.

\section{Material and methods}

Between 1993 and October of 2009, 463 patients with adrenal incidentalomas were diagnosed and treated in the Department of Endocrinology and Internal Diseases, Medical University of Gdansk as well as in the affiliated outpatient clinic. This group consisted of 325 female (70.2\%) and 138 male $(29.8 \%)$ patients aged 19 years to 83 years (mean age $57.8 \pm 12.7$ years). The patients were referred because of an adrenal tumour discovered during an ultrasound examination of the abdomen, performed for reasons other than a suspected adrenal lesion. The ultrasound findings were verified by abdominal computed tomography (CT) and/or magnetic resonance imaging $(\mathrm{MRI})$.

The following were regarded as indications for surgery: tumour size $\geq 4 \mathrm{~cm}$ (until 2004, $\geq 3 \mathrm{~cm}$ ), increasing tumour size during follow-up and/or abnormalities of adrenal hormonal function, or findings on imaging studies suggestive of malignancy. Several patients with adrenal masses smaller than $3 \mathrm{~cm}$ and without hormonal activity were referred for surgery because of their young age and severe anxiety.

Adrenalectomy was performed in 245 patients. The remaining 218 patients with smaller tumours without hormonal activity underwent periodic assessment of adrenal function and tumour size with CT (every 6-12 months). All patients are still being followed by the Endocrinology Outpatient Clinic (duration of follow-up 1 years to 15 years, mean duration 8 years).

\section{Hormonal assays and serum biochemistry}

In all patients, after obtaining detailed history, adrenocortical function was assessed by the determination of serum cortisol and dehydroepiandrosterone (DHEA) or dehydroepiandrosterone sulphate (DHEAS). Twenty-four-h urine was also collected for free cortisol, 17-ketosteroids (17-KS) and 17-hydroxysteroids (17-OHS). A $1 \mathrm{mg}$ overnight dexamethasone suppression test was also performed. The excretion of vanillyl mandelic acid (VMA) and/or metanephrines in 24-h urine was measured as indicators of adrenal medullary function. Serum electrolytes and glucose were checked in all patients. In all cases of hypokalaemia, urinary excretion of potassium was determined. Blood pressure and heart rate were also recorded.

Serum cortisol was measured at 8 a.m. and 8 p.m. in a stress-free environment, and blood samples were immediately delivered to the laboratory. The diagnosis of subclinical Cushing syndrome (SCS) was made when the concentration of cortisol after dexamethasone administration was $>140 \mathrm{nmol} / \mathrm{l}$. The above assessments were performed with the IMMULITE system by DPC. The morning serum DHEA and DHEAS concentrations were determined with a radioimmunological assay (RIA) using commercial DHEA/DHEAS kits by IMMUNOTECH and Orion Diagnostica, respectively. 24-h urinary cortisol was determined by RIA using commercial SPECTRIA Cortisol RIA kits by Orion Diagnostica. The colorimetric method (Zimmermann reaction with m-dinitrobenzene) was used for the measurement of urinary 17-ketosteroids. 17$\mathrm{OHS}$ were detected with the colorimetric method by Porter-Silber. Colour intensity was proportional to the concentration of hydroxysteroids. Vanillyl mandelic acid and metanephrines in 24-h urine were determined by RIA using commercial kits by Bio-Rad.

\section{Statistical analysis}

The measures of central tendency were compared using Studen's $t$ test for normally distributed variables (after logarithmic transformation if appropriate) or Mann-Whitney $U$ test for the remaining variables. The dependencies between nominal variables as well as contingency tables were analysed using Pearson's $\chi^{2}$ or Fisher's exact test. A twotailed significance level of $<0.05$ was regarded as statistically significant. All statistical analysis was undertaken with the Statistica 8.0 software (StatSoft, Inc., Tulsa, OK, USA; licensed for the Medical University of Gdansk).

\section{Results}

In the entire group of 463 patients, SCS was diagnosed in 31 (6.7\%) and subclinical pheochromocytoma (PHEO) in 37 patients (8.5\%). Not a single patient was diagnosed with subclinical Conn syndrome. Adrenocortical cancer was diagnosed in 12 surgically treated patients with incidentally discovered adrenal masses (4.7\%).

\section{The usefulness of urinary vanillyl mandelic acid and metanephrines in the diagnosis of pheochromocytoma}

Twenty-four-h urinary VMA excretion was measured preoperatively in 86 out of 245 surgically treated patients with incidentaloma, including 14 patients with PHEO. In PHEO patients, increased 24-h urinary excretion of VMA was significantly more fre- 
quent than in patients with other adrenal tumours $(p=0.012)$. The sensitivity of this parameter calculated in a group of 86 patients was $57 \%$, and specificity was $77 \%$ (Table I).

Twenty-four-h urinary excretion of metanephrines was measured preoperatively in 93 out of 245 surgically treated patients with incidentalomas, including 24 patients with PHEO. In patients with pheochromocytoma, increased concentration of metanephrines was more common than in patients with other pathological diagnoses. This difference was statistically significant $(p=0.001)$. The sensitivity of this parameter calculated in a group of 93 patients was $71 \%$, with $86 \%$ specificity (Table I).

\section{Serum concentration of DHEA/DHEA-S in benign and malignant adrenal lesions}

Out of 245 patients with surgically treated incidentalomas (including 25 malignant tumours: 12 adrenocortical cancers, 5 malignant pheochromocytomas and 8 adrenal metastases), the preoperative concentration of DHEAS was determined in 35 patients (30 female, 5 male) and that of DHEA in 143 patients (102 female, 41 male). In 97\% of patients with a benign adrenal mass, serum DHEA/DHEAS concentrations were within or below the age- and gender-specific reference range (low in $78 \%$ of cases, normal in $19 \%$ ). Increased concentrations of these hormones were noted in only $3 \%$ of benign lesions.

Serum DHEA/DHEAS concentrations exceeded the age- and gender-specific reference range in $25 \%$ of patients with malignant adrenal tumours, and were normal or low in $75 \%$ of cases (normal in 31\% of cases, low in $44 \%$ of cases). In all cases of adrenocortical carcinomas, the concentrations of DHEA/DHEAS were either normal or above the reference range. All patients with adrenal metastases had DHEA/DHEAS levels below the reference range. In conclusion, increased values of DHEA/DHEAS were more often found in malignant $(25 \%)$ than in benign tumours (3\%). This correlation was statistically significant $(p<0.01)$. Conversely, DHEA/DHEAS concentrations below the reference range were more common in patients with benign diseases (78\%) than those with malignancy (44\%). This correlation was also statistically significant $(p=0.017)$ (Table I).

\section{Urinary 17-ketosteroids in benign and malignant adrenal lesions}

Twenty-four-h urinary excretion of 17-ketosteroids was measured preoperatively in 164 out of 245 surgically treated patients with incidentaloma. The urinary excretion of 17-ketosteroids exceeded the upper reference limit in $91 \%$ of patients with adrenocortical carcinoma. In contrast, increased urinary excretion of 17-ketosteroids was found in only $58 \%$ of patients without the diagnosis of adrenocortical carcinoma. This difference was statistically significant $(p=0.02)$ (Table I).

\section{Morning serum cortisol after the administration of dexamethasone ("overnight" test) and urinary cortisol excretion and size of adrenal tumour}

Serum cortisol concentration in the dexamethasone test was measured in 314 out of 463 patients with incidentaloma. In the study group we found a relatively strong correlation between tumour size and the serum concentration of cortisol after the administration of dexamethasone $(r=0.42)$. This correlation was statistically significant ( $p<0.001$ ) (Figure 1). Similarly, in 373 out of 463 patients with incidentaloma, a strong correla-

Table I. Usefulness of various laboratory tests in clinical differentiation of adrenal tumours

\begin{tabular}{|c|c|c|c|}
\hline Urine VMA & Pheochromocytoma & Other adrenal tumours & Value of $p$ \\
\hline Elevated & $57.1 \%$ & $23.3 \%$ & 0.012 \\
\hline Normal & $42.9 \%$ & $76.7 \%$ & \\
\hline Urine methoxycatecholamines & Pheochromocytoma & Other adrenal tumours & Value of $p$ \\
\hline Elevated & $70.8 \%$ & $8.6 \%$ & $<0.001$ \\
\hline Normal & $29.2 \%$ & $91.4 \%$ & \\
\hline Serum DHEA(S) & Malignant tumours & Benign adrenal tumours & Value of $p$ \\
\hline Elevated & $25 \%$ & $3 \%$ & 0.017 \\
\hline Normal & $31 \%$ & $19 \%$ & \\
\hline Below normal & $44 \%$ & $78 \%$ & \\
\hline Urine 17-ketosteroids & Adrenocortical cancer & Other adrenal tumours & Value of $p$ \\
\hline Elevated & $91 \%$ & $58 \%$ & 0.020 \\
\hline Normal & $9 \%$ & $42 \%$ & \\
\hline
\end{tabular}


A

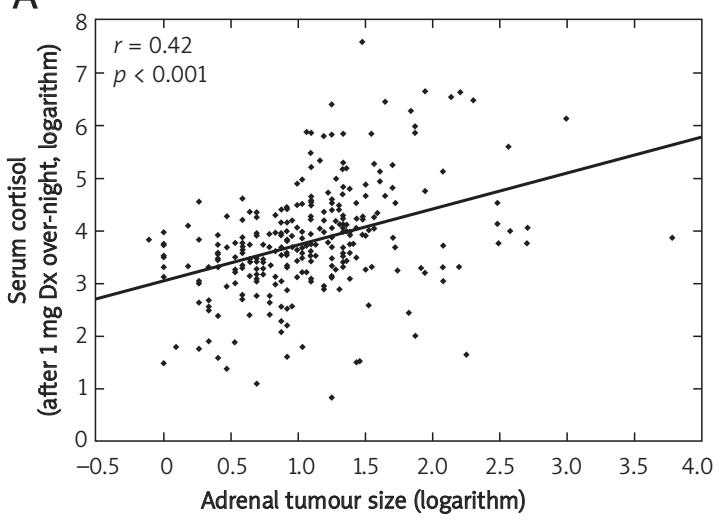

B

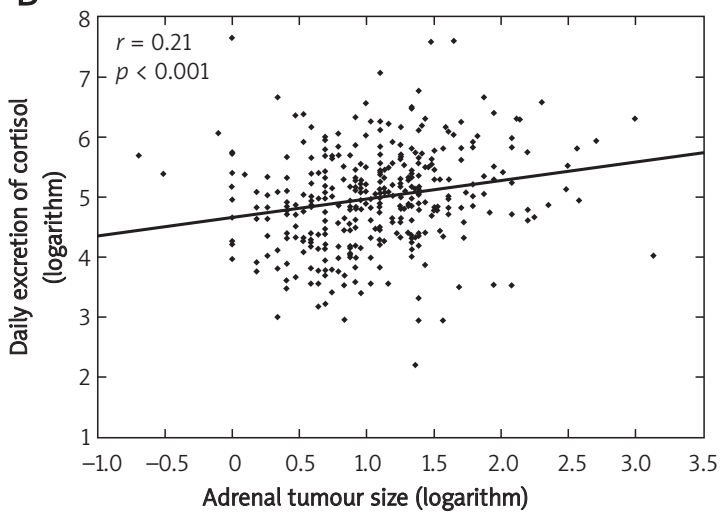

Figure 1. Correlations between adrenal tumour size and serum cortisol after $1 \mathrm{mg}$ of dexamethasone overnight (A) and urine daily excretion of cortisol (B)

tion was found between tumour size and the 24-h urinary excretion of cortisol. This correlation was also statistically significant $(p<0.001)$ (Figure 1$)$.

\section{Discussion}

The diagnostic work-up of adrenal incidentalomas is aimed at detection of malignancy (primary or metastatic) or subclinical hormonal hyperactivity. Establishing benign character of an incidentaloma provides reassurance to the patient and decreases the costs of follow-up studies. Therefore, many authors have tried to determine imaging and laboratory criteria for malignancy in adrenal incidentalomas $[2,4]$.

Tumours with biochemically confirmed increased secretion of cortisol, mineralocorticoids, androgens or catecholamines should be treated surgically. Thus, many authors recommend an array of hormonal assays in patients with adrenal incidentalomas. Most are not hormonally active but approximately $15 \%$ demonstrate hypersecretion of medullary or cortical hormones, especially with bilateral lesions [9].

Subclinical Cushing syndrome (SCS) is the most common hormonal abnormality, being found in $5 \%$ to $8 \%$ of patients with incidentalomas, according to different authors $[6,10]$.

Impaired suppression of cortisol secretion with dexamethasone occurs earlier than the increase in the urinary excretion of free cortisol and its metabolites. The low-dose dexamethasone suppression test, regarded as a screening test, is considered to be the best diagnostic parameter in SCS, yielding abnormal results in as many as $80 \%$ of patients [3]. According to most authors, the morning serum concentration of cortisol after dexamethasone administration above $140 \mathrm{nmol} / \mathrm{l}(>3 \mu \mathrm{g} / \mathrm{dl})$ permits the diagnosis of SCS $[3,4,11]$. Conversely, serum cortisol below $50 \mathrm{nmol} / \mathrm{l}(<1.8 \mu \mathrm{g} / \mathrm{dl})$ excludes subclinical as well as overt Cushing syndrome [11]. Concentrations between $50 \mathrm{nmol} / \mathrm{l}$ and $140 \mathrm{nmol} / \mathrm{l}$
(1-3 $\mu \mathrm{g} / \mathrm{dl})$ reflect low-level hormonal activity of the tumour [11]. Low serum adrenocorticotropic hormone $(\mathrm{ACTH})$ concentrations in the morning hours were observed in $78 \%$ of patients with SCS $[5,12]$. On the other hand, increased 24-h urinary excretion of cortisol was demonstrated in $64 \%$ of SCS cases, and low DHEAS levels in only $50 \%$ of cases $[9,13]$.

The frequency of SCS diagnosis depends on the number and sensitivity of hormonal assays employed to assess the function of the pituitaryadrenal axis. In the presented material, lack of suppression in the $1 \mathrm{mg}$ dexamethasone test was regarded as the most important parameter establishing the diagnosis of SCS. Subclinical Cushing syndrome was diagnosed if the morning serum cortisol concentration exceeded $140 \mathrm{nmol} / \mathrm{l}$. The diagnosis of SCS was made in 31 patients, making up $6.7 \%$ of all patients in whom the test was performed.

In some reports, increased 24-h urinary excretion of cortisol correlated positively with tumour size $[6,14]$. In our group of patients, this correlation was statistically significant $(p<0.001)$. Moreover, a statistical relationship was demonstrated between tumour size and the serum cortisol concentration assessed in the $1 \mathrm{mg}$ dexamethasone suppression test $(p<0.001)$.

Most cases of SCS are caused by an adenoma or bilateral adrenocortical hyperplasia [2, 9]. Adrenocortical carcinomas presenting as SCS or virilization syndrome have also been reported [7]. In our own material, out of 26 patients with preoperative diagnosis of SCS undergoing adrenalectomy (5 other patients with SCS were not treated surgically because of lack of consent, severe medical deterioration or advanced age), an adenoma of the adrenal cortex was diagnosed in $50 \%$ of cases $(13 / 26)$, adrenocortical carcinoma in $26.9 \%$ of cases $(7 / 26)$, nodular hyperplasia of the adrenal cortex in $15.4 \%$ of cases (4/26) and pheochromocytoma in $7.7 \%$ of 
cases (2/26). The granules of adrenomedullary cells secrete, besides catecholamines, several active peptides, including ACTH and corticotropin-releasing hormone $(\mathrm{CRH})$ [15]. Oversecretion of these may be responsible for the development of SCS in two of our patients with $\mathrm{PHEO}$.

The clinical signs accompanying hormonal changes in SCS are subtle or absent. Patients with SCS, more often than those without, are found to have hypertension ( 29 to $92 \%$ of cases), type 2 diabetes (14 to $42 \%$ of cases), obesity (43 to $67 \%$ of cases) and lipid abnormalities (28\% of cases) [16, 17]. The low-level secretion of glucocorticoids by adrenal incidentalomas can be a risk factor for cardiovascular disease and osteoporosis $[16,18]$. The reports discussed above demonstrate that SCS is not entirely asymptomatic $[12,16]$. In our group of patients with incidentalomas, we assessed the prevalence of concomitant type 2 diabetes and hypertension, but the results were not statistically significant.

The possible development of a clinically symptomatic Cushing syndrome determines further management of patients with SCS. According to some authors, the likelihood of such development can be as high as 17\% [19]. In contrast, in a study by Libe, none of the 12 patients with SCS developed overt CS during 25.5 months of follow-up [20]. At the same time, a gradual increase in hormonal activity, even in the absence of recognized criteria for CS, can be a sign of progression towards this condition [20]. In our own material, none of the 5 patients with the diagnosis of PCS who did not undergo adrenalectomy developed clinically symptomatic Cushing syndrome.

A previously hormonally inactive tumour may, over time, demonstrate subclinical hypersecretion of cortisol, fulfilling the criteria for PCS [19]. In our study group, this transition occurred in two cases. In both of these patients, signs of progression in the hormonal assays were associated with increasing tumour size on imaging studies. Both patients were referred for surgery.

Among patients with incidentalomas and subclinical Conn syndrome, hypertension was reported in $1.5-3.3 \%$ of cases $[11,13,21]$. In this group, beside hypertension, the remarkable finding was the borderline low potassium concentration in $60 \%$ up to $84 \%$ of cases $[11,13,21]$. On the other hand, normokalaemia is found in $7 \%$ to $38 \%$ of patients with Conn syndrome. This phenomenon is usually explained by sodium depletion leading to potassium retention, minimizing hypokalaemia [13].

Excellent diagnostic tests are the serum aldosterone concentration and serum renin activity (SRA), both at baseline and after stimulation [15]. In our study, while the serum potassium concentration was normal in all cases, aldosterone and SRA were not measured. This is probably the reason why no cases of subclinical hyperaldosteronism were diagnosed.

The likelihood of PHEO in a patient with adrenal incidentaloma is low, at $1.5 \%$ to $11 \%$ [15]. On the other hand, as many as $10-20 \%$ of these tumours are detected incidentally $[9,15]$. In our group of patients with incidentaloma, pheochromocytoma was diagnosed in $8.5 \%$ of cases. Pheochromocytomas are characterised by variable hormonal activity, depending on their size. Small tumours are usually more active, which translates into secreting larger amounts of catecholamines into the blood stream. Based on our material, no statistically significant correlation was found between the size of a PHEO and the 24-h urinary secretion of VMA and metanephrines.

The serum concentration of metanephrines has been found to correlate with the overproduction of catecholamines in tumour tissue $[11,15]$. Another very useful and widely available test is the measurement of 24-h urinary metanephrines (sensitivity $86 \%$, specificity $97 \%$ ). The widely used assessment of 24-h urinary VMA should not be recommended in suspected $\mathrm{PHEO}$ because of the low sensitivity (under 50\%) [3]. In our material, the sensitivity of VMA assessment in the diagnosis of PHEO was $57 \%$, with $77 \%$ specificity. These values were similar to those found in the current literature. On the other hand, the sensitivity of determination of 24-h urinary metanephrines was $71 \%$, with $86 \%$ specificity - values only slightly lower than those reported in the literature [13].

Many studies show that 3\% to 10\% (seldom $20 \%$ ) of all PHEOs prove to be malignant $[15,22]$. In our material, malignant PHEOs (based on the presence of distant metastases) were diagnosed in $13.5 \%$ of cases (5/37). The slightly higher, compared to data from the literature, percentage of patients with malignant tumours can probably be explained by the relatively small number of patients. It should be stressed that there are no definite histopathological criteria for malignant PHEO, and the diagnosis is based on the presence of distant metastases in organs that do not normally contain chromaffin tissue. Hypertension was more common in patients with benign pheochromocytomas compared with malignant PHEOs; this difference was statistically significant $(p=0.02)$. Patients with malignant PHEOs are more often found to have increased dihydroxyphenylalanine (DOPA) levels, and it is the hypotensive effect of DOPA that is regarded as the possible explanation of normal blood pressures in this subset of patients.

Carcinoma of the adrenal cortex is found with varying frequency in patients with adrenal incidentalomas (usually 5\%) and it more commonly affects females [23]. Nearly $50 \%$ of incidentally discovered carcinomas of the adrenal cortex are not 
hormonally active. In hormonally active adrenocortical carcinomas, the concomitant secretion of both cortisol and androgens has been observed [6, 23]. Carcinomas of the adrenal cortex are usually large tumours, measuring over $4 \mathrm{~cm}$ in diameter. In $20 \%$ to $50 \%$ of cases, distant metastases are already present at the time of diagnosis [2, 6, 23, 24]. Undoubtedly, the size of the adrenal incidentaloma is a risk factor for the presence of malignancy, but size alone does not absolutely determine its character.

The large production of DHEAS due to hypertrophy of the zona reticularis can often suggest the diagnosis of adrenocortical carcinoma. The concentration of DHEA/DHEAS is increased or at the upper limit of normal in more than $90 \%$ of cases of adrenocortical carcinoma and is, beside the urinary 17-ketosteroids, the second sensitive marker of this neoplasm [14]. Terzolo et al. demonstrated that low (41\%) or normal (59\%) serum concentrations of DHEAS were associated with the pathological diagnosis of a benign adrenal lesion [12]. On the other hand, results obtained by Bencsik et al. were less promising [8]. In as many as half of 12 patients with malignant neoplasms of the adrenal glands, the concentrations of DHEAS were below the reference range. In our own material, high concentrations of DHEA/DHEAS were more often found in malignant (25\%) than benign (3\%) adrenal tumours. This difference was statistically significant $(p<0.01)$. In all cases of adrenocortical carcinomas, the concentrations of DHEA/DHEAS were either normal or above the reference range. Conversely, concentrations of DHEA/DHEAS below the reference range were associated with a benign lesion in $78 \%$ of cases. The usefulness of DHEAS is limited by the difficulty in distinguishing lower concentrations due to a benign tumour or due to the physiologically decreased function of the gland in the elderly.

In cases of subclinical androgen excess that may accompany accidentally discovered adrenocortical carcinomas, increased excretion of androgen metabolites in the 24-h urine is typical. Increased urinary 17-ketosteroids, alongside increased serum androgens, are a sensitive marker for this neoplasm $[1,2]$. In our own material, 17-ketosteroids exceeded the upper reference limit in $91 \%$ of patients with adrenocortical carcinoma. On the other hand, only $58 \%$ of patients with pathologically confirmed benign lesions had concentrations of 17-ketosteroids above the upper limit of normal. This difference was statistically significant $(p=0.02)$.

Most authors suggest that the prognosis in adrenocortical carcinomas does not depend on their hormonal activity. Others believe that the presence of hormonal activity improves survival in this type of cancer [6, 23]. Among our patients treated sur- gically for adrenocortical cancer, 12 had incidentalomas (4.7\%). Most patients had subclinical hormonal activity in the form of SCS (26.9\%) and increased adrenal androgen production as determined by the level of urinary 17-ketosteroids (91\%). Five patients died within a few months after diagnosis, 4 are being treated with mitotane, and 3 were lost to follow-up.

Malignant adrenal tumours carry a poor prognosis. The 5 -year survival rate in adrenocortical carcinoma ranges from $32 \%$ to $45 \%[6,23]$, whereas the mean survival in malignant PHEOs is only 28 months [25]. Thus, early diagnosis and appropriate treatment increase the patient's chance of cure.

Further research in large groups of patients can help establish new prognostic parameters and the optimal management of these tumours.

In conclusion, clinically "silent" incidentalomas are often associated with subclinical hormonal activity (subclinical Cushing's syndrome or subclinical pheochromocytoma). Such patients should undergo surgery regardless of tumour size.

High urinary concentrations of 17-ketosteroids correlate positively with the diagnosis of adrenocortical carcinoma.

High serum concentrations of DHEA/DHEAS correlate positively with the diagnosis of a malignant adrenal tumour, whereas DHEA/DHEAS concentrations below the age- and gender-specific reference range are more common in patients with benign diseases.

\section{References}

1. Kasperlik-Załuska AA, Otto M, Cichocki A, et al. Incidentally discovered adrenal tumors: a lesson from observation of 1,444 patients. Horm Metab Res 2008; 40: 338-41.

2. Sworczak K, Babińska A, Stanek A, et al. Clinical and histopathological evaluation of the adrenal incidentaloma. Neoplasma 2001; 48: 221-6.

3. Mantero F, Arnaldi G. Management approaches to adrenal incidentalomas. A view from Ancona, Italy. Endocrinol Metab Clin North Am 2000; 29: 107-25.

4. Barzon L, Scaroni C, Sonino N, Fallo F, Paoletta A, Boscaro M. Risk factors and long-term follow-up of adrenal incidentalomas. J Clin Endocrinol Metab 1999; 84: 520-6.

5. Reincke M, Nieke J, Krestin GP, Saeger W, Allolio B, Winkelmann W. Preclinical Cushing's syndrome in adrenal "incidentalomas": comparison with adrenal Cushing's syndrome. J Clin Endocrinol Metab 1992; 75: 826-32.

6. Kasperlik-Załuska AA, Otto M, Cichocki A, et al. 1,161 patients with adrenal incidentalomas: indictions for surgery. Langenbecks Arch Surg 2008; 393: 121-6.

7. Höfle G, Gasser RW, Lhotta K, Janetschek G, Kreczy A, Finkenstedt G. Adrenocortical carcinoma evolving after diagnosis of preclinical Cushing's syndrome in an adrenal incidentaloma. A case report. Horm Res 1998; 50: 237-42.

8. Bencsik Z, Szabolcs I, Kovacs Z, et al. Low dehydroepiandrosterone sulfate (DHEA-S) level is not a good 
predictor of hormonal activity in nonselected patients with incidentally detected adrenal tumors. J Clin Endocrinol Metab 1996; 81: 1726-9.

9. Barzon L, Sonino N, Fallo F, Palu G, Boscaro M. Prevalence and natural history of adrenal incidentalomas. Eur J Endocrinol 2003; 149: 273-85.

10. Young WF Jr. Management approaches to adrenal incidentalomas: a view from Rochester, Minnesota. Endocrinol Metab Clin North Am 2000; 29: 159-85.

11. Grumbach MM, Biller BM, Braunstein GD, et al. Management of the clinically inapparent adrenal mass ("incidentaloma"). Ann Intern Med 2003; 138: 424-9.

12. Terzolo M, Osella G, Ali A, et al. Subclinical Cushing's syndrome in adrenal incidentaloma. Clin Endocrinol (Oxf) 1998; 48: 89-97.

13. Mantero F, Masini AM, Opocher G, Giovagnetti M, Arnaldi G. Adrenal incidentaloma: an overview of hormonal data from the National Italian Study Group. Horm Res 1997; 47: 284-9.

14. Terzolo M, Osella G, Ali A, et al. Different patterns of steroid secretion in patient with adrenal incidentaloma. J Clin Endocrinol Metab 1996; 81: 740-4.

15. Bravo EL, Tagle R. Pheochromocytoma: state-of-the-art and future prospects. Endocr Rev 2003; 24: 539-53.

16. Tauchmanova L, Rossi R, Biondi B, et al. Patients with subclinical Cushing's syndrome due to adrenal adenoma have increased cardiovascular risk. J Clin Endocrinol Metab 2002; 87: 4872-8.

17. Torlontano M, Zingrillo M, D'Aloiso L, et al. Pre-Cushing's syndrome not recognized by conventional dexamethasone suppression-tests in an adrenal "incidentaloma" patient. J Endocrinol Invest 1997; 20: 501-4.

18. Tsagarakis S, Roboti C, Kokkoris P, Vasiliou V, Alevizaki C, Thalassinos D. Elevated post-dexamethasone suppression cortisol concentrations correlate with hormonal alterations of the hypothalamo-pituitary adrenal axis in patients with adrenal incidentalomas. Clin Endocrinol (Oxf) 1998; 49: 165-71.

19. Barzon L, Fallo F, Sonino N, Boscaro M. Development of overt Cushing's syndrome in patients with adrenal incidentaloma. Eur J Endocrinol 2002; 146: 61-6.

20. Libe R, Dall'Asta C, Barbetta L, Baccarelli A, Beck-Peccoz P, Ambrosi B. Long-term follow-up study of patients with adrenal incidentalomas. Eur J Endocrinol 2002; 147: 489-94.

21. Bernini G, Moretti A, Argenio G, Salvetti A. Primary aldosteronism in normokalemic patients with adrenal incidentalomas. Eur J Endocrinol 2002; 146: 523-9.

22. Sworczak K, Zaczek A, Babinska A, Lisowska U, Bielawski KP, Falkiewicz B. Gene copy numbers of erbB oncogenes in human pheochromocytoma. Oncol Rep 2002; 9: 1373-8.

23. Libe R, Fratticci A, Bertherat J. Adrenocortical cancer: pathophysiology and clinical management. Endocr Relat Cancer 2007; 14: 13-28.

24. Babinska A, Sworczak K, Wisniewski P, Nałecz A, Jaskiewicz K. The role of immmunohistochemistry in histopathological diagnostics of clinically „,silent” incidentally detected adrenal masses. Exp Clin Endocrinol Diabetes 2007; 116: 246-51.

25. Kopf D, Goretzki PE, Lehnert H. Clinical management of malignant adrenal tumors. J Cancer Res Clin Oncol 2001; 127: 143-55. 